\title{
Integrated Cryogenic and Thermal Energy Storage for Decarbonizing Energy Consumption: Development and Challenges
}

\author{
María Elena Navarro, ${ }^{1}$ Abdalqader Ahmad, ${ }^{1}$ Yimo Luo ${ }^{2}$ and Xiaohui She ${ }^{1, *}$
}

Accepted 28 January 2019

DOI: $10.30919 / \mathrm{esee} 8 \mathrm{c} 300$

\section{Introduction}

Global warming promotes a profound transformation from fossil fuel to renewable energy in key sectors such as heat, transport and power. It is reported that there is an absolute increase of $\sim 0.2 \%$ per year in the share of renewable energy in the total final energy consumption in recent years. However, to keep the global temperature rise well below $2{ }^{\circ} \mathrm{C}$, as stated in the Paris Agreement, the renewable energy share needs to be raised from $19 \%$ in 2017 to $65 \%$ by 2050 , indicating an annual increase $\sim 7$ times larger than it has been. ${ }^{1}$ With the rapid development of renewable energy, its intermittency nature becomes a rising concern, which not only brings a mismatch between energy supply and demand, but also causes safety issues to the power grid. In this context, energy storage can act as a buffer to compensate the intermittency by storing energy at off-peak times and releasing it at peak times.

Among large-scale energy storage technologies (pumped hydro, compressed air, etc.), cryogenic energy storage is the most promising due to the advantages in terms of ambient pressure storage (i.e. no geographical constraints), large energy storage density $(60-120 \mathrm{Wh} / \mathrm{L})$, $100 \%$ discharging, fast response ( 2 mins), etc. Moreover, the synergy of using a combination of thermal energy storage and cryogenic energy storage allows the hybrid system to achieve a better performance at the cost of higher complexity.

\section{Cryogenic Energy Storage}

Cryogenic energy storage (CES) uses liquid air or liquid nitrogen as energy storage media, hence also known as Liquid Air Energy Storage. The basic working principle of the CES is shown in Fig. 1, which includes air liquefaction and power recovery processes. In the air liquefaction process, the ambient air is firstly purified to remove $\mathrm{CO}_{2}$ and water; then, it is compressed to a high pressure by consuming offpeak electricity or renewable energy, and meanwhile the heat of compression is stored in the heat storage tank for later use in the power recovery process; the high pressure air is cooled down by cold energy from the cold storage tank, and then expands to ambient pressure for generating liquid air.

In the power recovery process, the liquid air is pumped to a high pressure, releasing the cold energy into the cold storage tank for later

'Birmingham Centre for Energy Storage, School of Chemical Engineering, University of Birmingham, Birmingham, B15 2TT, UK

${ }^{2}$ Shenzhen Institute, Peking University, Shenzhen 518055, China

*E-mail: X.She@bham.ac.uk use in the air liquefaction process; then, it is heated by the heat of compression from the heat storage tank and finally expands in air turbines to generate peak and stable electricity. Round trip efficiency is usually used to assess the thermodynamic performance of the CES, which is defined as the ratio of power generation during power recovery to power consumption during air liquefaction. It is clear from Fig. 1 that the CES is closely integrated with thermal energy storage.

\subsection{Large-scale Cryogenic Energy Storage for Power Network}

The large-scale CES was firstly proposed for peak-shaving of power network by Smith from University of Newcastle upon Tyne in $1977,{ }^{2}$ as shown in Fig. 2a. Since then, substantial progress was made due to the collaboration between Highview Power Storage and University of Leeds from 2005, and subsequently the first pilot plant of CES (350 $\mathrm{kW} / 2.5 \mathrm{MWh}$ ) was designed and built in the period of 2009-2012, as shown in Fig. 2b. It was later donated to University of Birmingham for further research in 2013. The pilot plant has a low round trip efficiency of $\sim 12 \%$, which is mainly due to the small plant size and lack of thermal storage. In late 2018, a pre-commercial CES plant (5 MW/15 MWh) was developed by Highview Power Storage (see Fig. 2c), which was reported to have a higher round trip efficiency of $\sim 60 \%$. A number of studies have been conducted to further enhance the round trip efficiency of the CES especially since 2013, including packed bed cold and heat storage, ${ }^{3,4}$ effective cold and heat utilization, ${ }^{5,6}$ integration with industrial waste cold and heat energy, ${ }^{7,8}$ etc.

It is clear that thermal energy storage plays a key role to improve the round trip efficiency of the CES. However, both the current academic research and industrial application mainly focus on sensible heat storage with pebbles or thermal fluids, which has some drawbacks such as a low energy storage efficiency or a low flammability point, respectively. Therefore, modified pebbles with a high heat capacity and thermal conductivity are required, and measures should be taken to improve the flammability points of thermal fluids for safety issues. In addition, other low-cost and large energy storage density technologies, such as latent or thermal-chemical energy storage, should be investigated for the CES in future studies. Further, the CES has a relatively higher capital cost and correspondingly a longer payback period of $\sim 10$ years. ${ }^{9}$ To enhance its economic competitiveness, multifunctional CES is required to develop for gaining more benefits besides peak-shaving, such as frequency regulation and black start of power network.

2.2 Small-scale Cryogenic Energy Storage for Zero-emission Vehicles The early attempt to use liquid nitrogen for driving vehicles appeared in 
1900 in the USA by Tripler Liquid Air Company. In 1973, Volkswagen Beetle $^{10}$ firstly developed a prototype of liquid nitrogen car where a liquid nitrogen dewar was fitted in a car to provide high pressure liquid to a heat exchanger before introducing it to a vane-type pneumatic motor to generate power, as shown in Fig. 2d. A more efficient system of using liquid nitrogen was developed by Peter Dearman in 2001, ${ }^{11}$ as shown in Fig. 2e. In this system, liquid nitrogen was pumped to high pressure and then passed through a heat exchanger to be evaporated and superheated; finally, the high pressure nitrogen drove a reciprocating engine to generate power. His novel idea was to spray an anti-freezing fluid into the engine during the air expansion process to maintain the air temperature constant (isothermal expansion). The early prototypes of this engine were built and fitted to small cars that ran with a speed of $30 \mathrm{mph}$. The most recent prototypes of this system were built by Dearmancompany in 2013 and fitted on a food transport truck for commercialization in 2017, as shown in Fig. 2f. In this system, the liquid nitrogen produces power to drive the conventional transport refrigeration unit rather than the car; liquid nitrogen before expansion provides extra cold energy to the cooling space, and the exhaust cold energy from the circulated anti-freezing fluid is used to enhance the conventional refrigeration unit. ${ }^{12}$

The current available systems use liquid nitrogen instead of liquid air as the fuel for vehicles, even though using liquid air can save up to $20 \%$ of the energy compared with liquid nitrogen. ${ }^{13}$ There are two main reasons: (a) liquid air is not commercially available due to its very limited demands and applications; (b) safety issues, such as differential boil off of liquid air, during the storage and transport processes (which is evaporation of the liquid air components at different rates leaving enrichment of oxygen concentration in the remaining liquid or gases in the system). There are different techniques to overcome these challenges such as having a cold head fitted on the top of the storage tank to condense the evaporated gases and return them back to the liquid bulk, but it is not cost effective. ${ }^{14}$ A more promising technique is the passive cold head which is still under investigation. ${ }^{15}$

\section{Thermal Energy Storage}

Cryogenic energy storage (CES) is closely integrated with Thermal Energy Storage (TES), as shown in Fig. 1. The development of the TES benefits the CES. TES covers a range of technologies based on exploiting different fundamental scientific principles. It can be classified into three groups: sensible heat storage, latent heat storage and thermochemical heat storage. Comparisons of the three groups are shown in Fig. 3. Sensible heat storage is the most commonly deployed and commercialized, which stores the energy by heating or cooling storage media (liquid or solid). The stored energy depends on the temperature gradient and the media properties. The working temperature range can go from $-196{ }^{\circ} \mathrm{C}$ to more than $1000{ }^{\circ} \mathrm{C} .{ }^{16}$ Latent heat storage uses phase change materials (PCMs), which undergo a phase transition to store the thermal energy. The energy stored depends on the latent heat of the PCMs and the key criterion for its selection is the phase change temperature, which has to meet the application need. Besides the phase change temperature, the latent heat of fusion and thermal conductivity are also considered to optimize the performance of the process. Thermochemical heat storage (THS) are the most compact systems. ${ }^{17}$ The THS materials benefit from the use of the energy involved in a chemical reaction, an adsorption or an absorption process. This technology performance is determined by the kinetics of the reaction and the system design. It has low heat losses, small storage volume $^{18}$ and the potential for long term storage as well as the ability to transport thermal energy for long distances. THS materials have a specific energy that is approximately 10 times higher than sensible heat storage, and 2 times higher than PCMs. However, THS systems are not yet commercialized, thus research and development are required to better understand and design these technologies. ${ }^{19}$

The current key barriers of thermal energy storage technologies, to act as key enablers for energy transition, depend on not only technological innovation needs but also each country needs and power system development stages. Therefore, a specific and appropriate whole-system approach has to be followed to accelerate its deployment. Among the technological barriers for TES development, the primary focus and efforts on the electrification of transport has set aside heat. This lack of focus has turned into a low number of demonstration projects across all TES technologies and markets. Demonstration projects are essential to encourage investment on the different sectors and show companies evidence of installation, operation and maintenance costs. Additionally, the rise of awareness of TES technologies will help to increase the public acceptability and market demand.

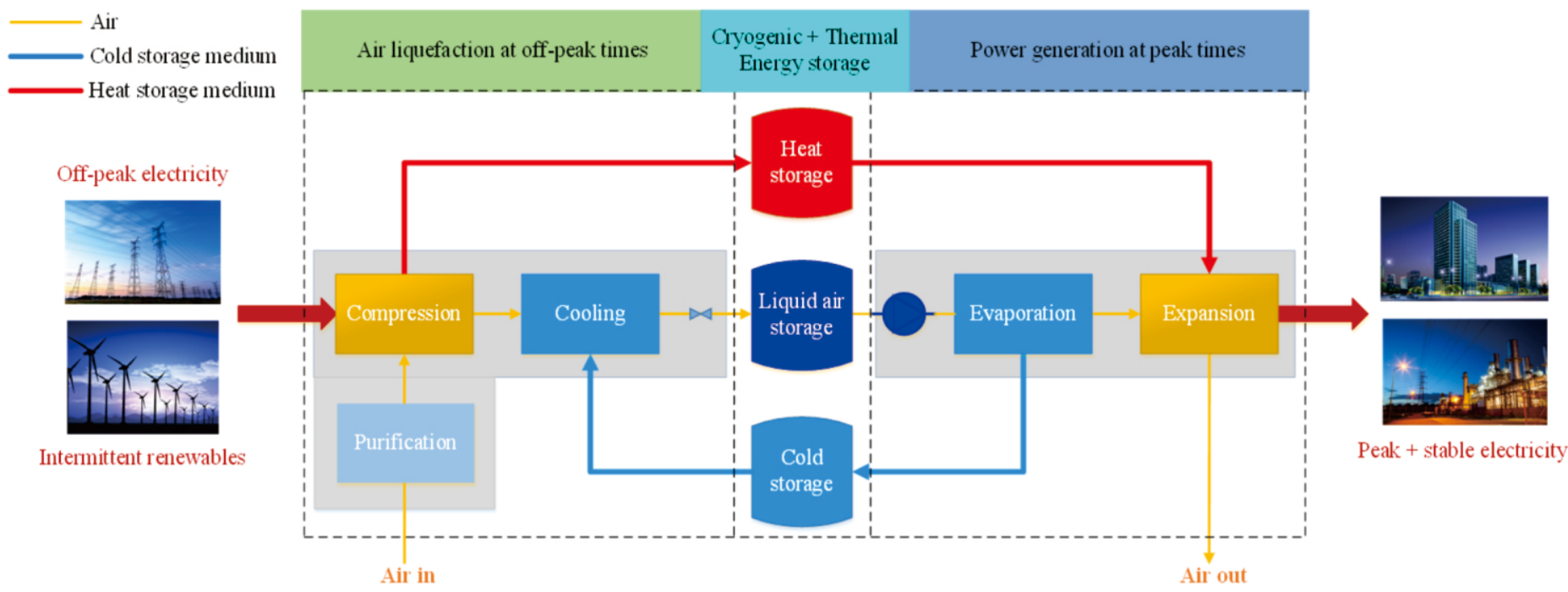

Fig. 1 Basic working principle of the cryogenic energy storage. 

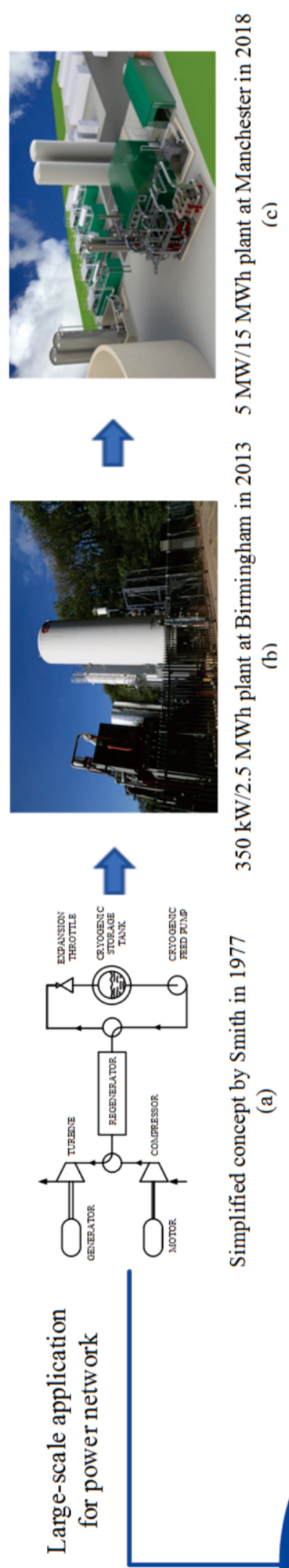

홍
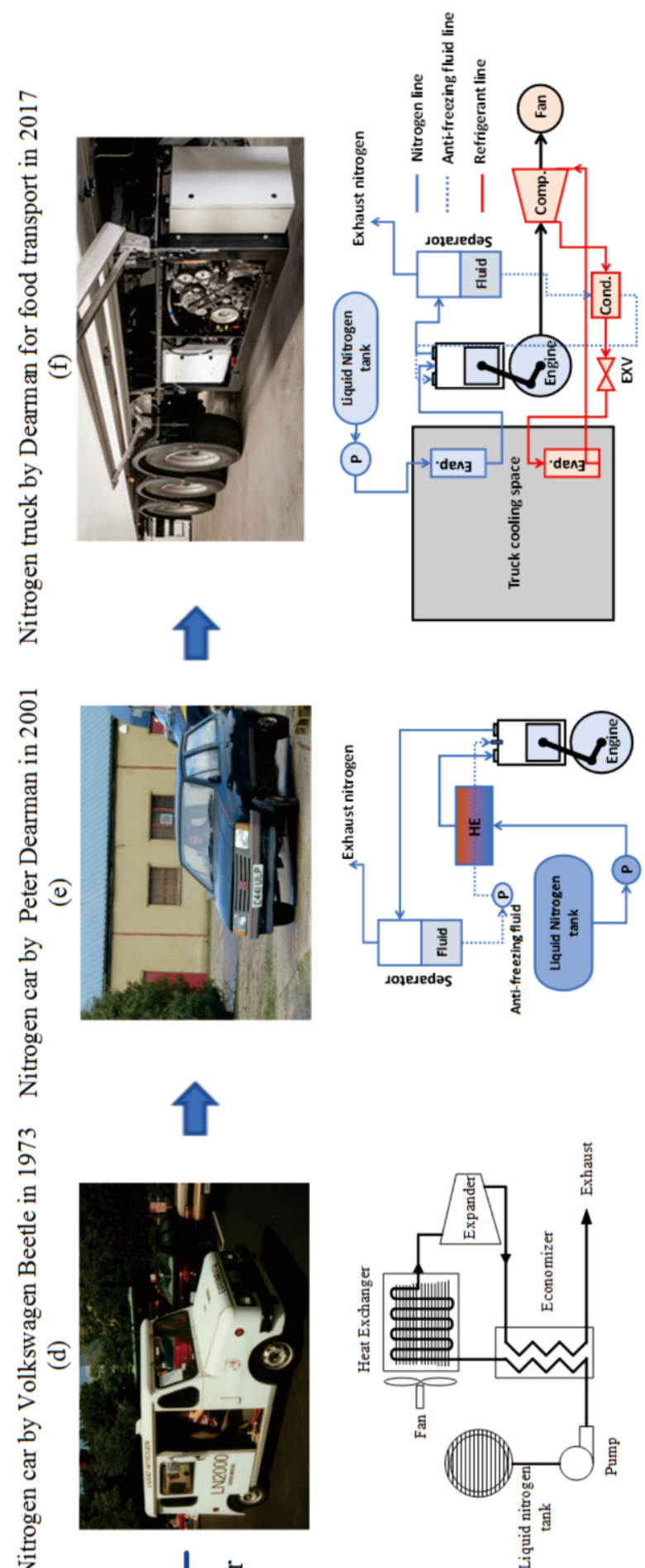

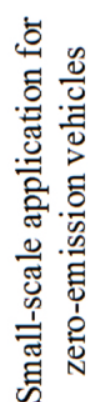
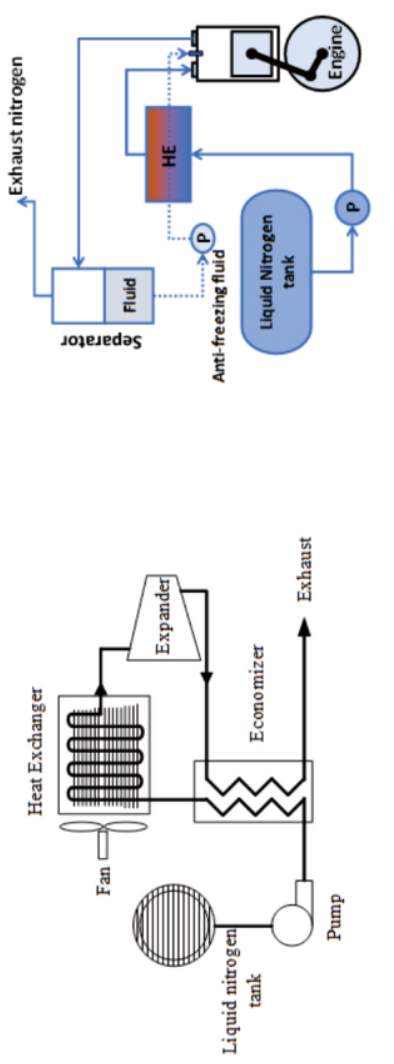

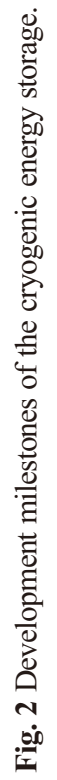

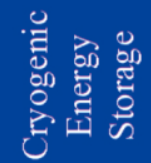




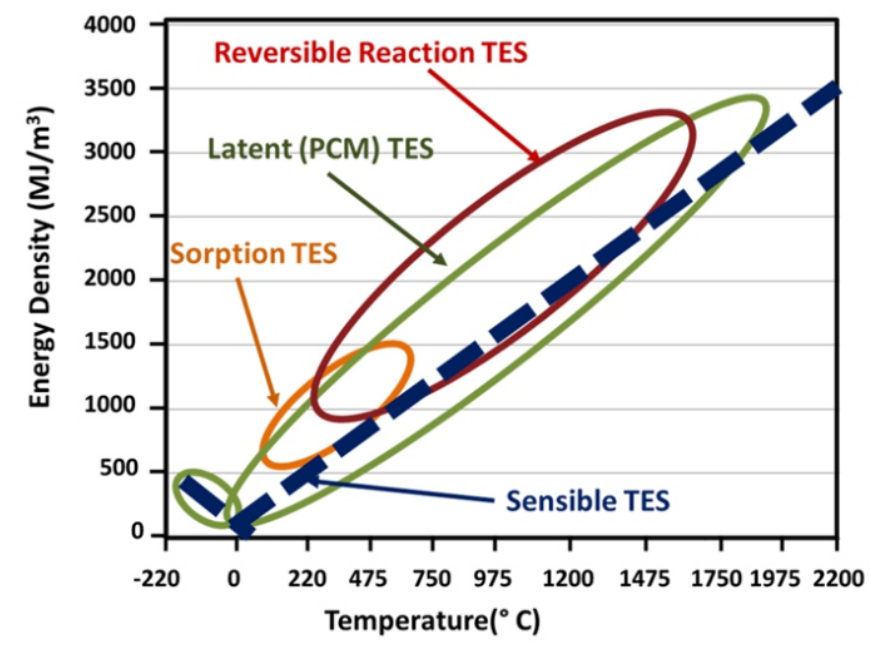

Fig. 3 Comparisons of the thermal energy storage technologies.

\section{Concluding remarks}

Cryogenic energy storage (CES) is one of the most promising technologies for tackling climate change. It is not only applicable for decarbonising power networks but also for zero-emission vehicles. The development of the CES is closely related to thermal energy storage (TES). Advanced TES technologies are needed for improving the CES round trip efficiency. In addition, it is necessary to develop multifunctional CES to enhance its economic benefits, such as frequency regulation and black start, besides arbitrage.

\section{Acknowledgements}

The authors are grateful for the partial support from a GCRF Collaborative Research Design Sandpits grant (University of Birmingham) and National Natural Science Foundation of China (Project No. 51706003).

\section{References}

1. International Renewable Energy Agency, Global Energy Transformation: A Roadmap to 2050. 2018.

2. E. M. Smith. Storage of electrical energy using supercritical liquid air. ARCHIVE: Proceedings of the Institution of Mechanical Engineers 18471982, 1977, 191, 89-98.

3. A. Sciacovelli, A. Vecchi and Y. L. Ding, Appl. Energy, 2017, 190, 84-98.

4. H. Peng, X. Shan, Y. Yang and X. Ling, Appl. Energy, 2018, 211, 126-35.

5. X. She, X. Peng, B. Nie, G. Leng, X. Zhang, L. Weng, L. Tong, L. Zheng, L. Wang and Y. Ding, Appl. Energy, 2017, 206, 1632-42.

6. X. Peng, X. She, L. Cong, T. Zhang, C. Li, Y. Li, L. Wang, L. Tong and Y. Ding, Appl. Energy, 2018, 221, 86-99.

7. Y. L. Li, H. Cao, S. Wang, Y. Jin, D. Li, X. Wang and Y. Ding, Appl. Energy, 2014, 113(1), 1710-1716.

8. I. Lee, J. Park and I. Moon, Energy, 2017, 140, 106-15

9. C. Xie, Y. Hong, Y. Ding, Y. Li and J. Radcliffe, Appl. Energy, 2018, 225, 244-257.

10. M. E. Parker, M. C. Plummer and C. A. Ordonez. CooLN2Car: An Experimental Car Which Uses Liquid Nitrogen as Its Fuel. 1997, In APS Texas Sections Fall Meeting Abstracts.

11. The potential of air - born out of a garage https://birminghamenergyinstitute.org /2016/05/31/the-potential-of-air-born-out-of-a-garage-invention-liquid-air-intotwo-commercial-demonstrations/

12. Dearman, Liquid Air on the European Highway. The economic and environmental impact of zero-emission transport refrigeration online access 30/12/2018.

13. A. Ahmad, R. Al-Dadah and S. Mahmoud, J. Clean Prod., 2017, 149, $773-$ 783.

14. D. W. Plachta, M. C. Guzik, Cryogenics, 2014, 60, 62-67.

15. R. C. Lee, LindeInc, Apparatus for storing a multi-component cryogenic liquid.U.S. Patent 5, 1996, 571, 231.

16. Ease/Eera, "EASE/EERA Technology Development Roadmap Update 2017," 2012, 108

17. A. H. Abedin, Renew. Energy, 2011, 4, 42-46.

18. D. Aydin, S. P. Casey and S. Riffat, Renew. Sust. Energ. Rev, 2015, 41, 356367.

19. N. Yu, R. Z. Wang, and L. W. Wang, Prog. Energy Combust. Sci., 2013, 39(5), 489-514.

Publisher's Note Engineered Science Publisher remains neutral with regard to jurisdictional claims in published maps and institutional affiliations. 